\title{
FIRST RESULTS OF THE ROSEBUD DARK MATTER EXPERIMENT
}

\author{
S. Cebrián ${ }^{a}$, N. Coron ${ }^{b}$, G. Dambier ${ }^{b}$, E. García $^{a}$, D. González ${ }^{a}$, \\ I.G. Irastorza ${ }^{a}$, J. Leblanc ${ }^{b}$, P. de Marcillac ${ }^{b}$, A. Morales ${ }^{a}$, \\ J. Morales ${ }^{a}$, A. Ortiz de Solórzano ${ }^{a}$, A. Peruzzi ${ }^{a}$, \\ J. Puimedón ${ }^{a}$, M.L. Sarsa ${ }^{a}$, S. Scopel ${ }^{a}$, J.A. Villar ${ }^{a}$ \\ ${ }^{a}$ Laboratorio de Física Nuclear y Altas Energías, \\ Universidad de Zaragoza, 50009 Zaragoza, Spain \\ ${ }^{b}$ Institut d'Astrophysique Spatiale, Bât. 121, \\ 91405 Orsay Cedex, France
}

\begin{abstract}
ROSEBUD (Rare Objects SEarch with Bolometers UndergrounD) is an experiment which attempts to detect low mass Weak Interacting Massive Particles (WIMPs) through their elastic scattering off $\mathrm{Al}$ and $\mathrm{O}$ nuclei. It consists of three small sapphire bolometers (of a total mass of $100 \mathrm{~g}$ ) with NTD-Ge sensors in a dilution refrigerator operating at $20 \mathrm{mK}$ in the Canfranc Underground Laboratory. We report in this paper the results of several runs (of about 10 days each) with successively improved energy thresholds, and the progressive background reduction obtained by improvement of the radiopurity of the components and subsequent modifications in the experimental assembly, including the addition of old lead shields. Mid-term plans and perspectives of the experiment are also presented.
\end{abstract}

PACS: 95.35.+d; 07.57.Kp; 07.62.+s

Keywords: Dark matter; WIMPs; Underground detectors; Bolometers

\section{Introduction}

Among the particle candidates to the non-baryonic dark matter of the universe, the neutral, weak interacting massive particles (WIMPs) supposedly forming a significant part of the galactic haloes - enjoy a prominent position. A distinguished WIMP is the neutralino, the lightest stable particle of the minimal supersymmetric extension of the Standard Model 1 . Accelerator results settle a lower bound of $20-30 \mathrm{GeV}$ to the neutralino mass in most of the models, but in unconstrained SUSY models masses as low as $2 \mathrm{GeV}$ could be allowed. WIMPs of $\mathrm{m} \sim 1-1000$ $\mathrm{GeV}$ with non-relativistic velocity, can interact with the nuclei of a detector target producing a nuclear recoil of a few $\mathrm{keV}$, a fraction of which is visible in the detector (depending on the nuclear target, the detector 
and the mechanism of energy deposition). Because of the low interaction rate and small energy deposition the WIMP detectors require very low backgrounds and energy thresholds 3 . A further contribution to the direct detection rate - in the very low energy region $\leq 1 \mathrm{keV}$ - could be provided by a non-thermal, low velocity WIMP population recently proposed 6 and discussed 5

Thermal detectors 6 , which measure the temperature increase produced by the WIMP interaction in an absorber crystal, use more efficiently the energy deposition of WIMPs than the conventional ionization detectors because most of the nuclear recoil energy in WIMP scattering goes to heat. They are true low energy detectors, where the visible energy is practically the whole recoil energy (quenching factor close to one). Moreover, the mechanisms and quanta involved in the physics of the detection imply that they should have better energy threshold and energy resolution than the conventional ionization detectors. In particular, the energy resolution on nuclear recoils achieved by the sapphire bolometers of the experiment reported in this paper is significantly better than that obtained with HPGe diodes, like COSME (which features a full width at half maximum (FWHM) energy resolution $\Gamma(10 \mathrm{keV})=0.4 \mathrm{keV})$. In much the same way the energy threshold of the sapphire bolometers of this worfinglso lower than that obtained in Ge-diodes looking for dark matter 3. 8. 6. Such low energy thresholds, hopefully achieved by ther mal detectors, make them valuable tools in the search for phenomenat leaving very small energy in the absorbers. So the cryogenic detectors

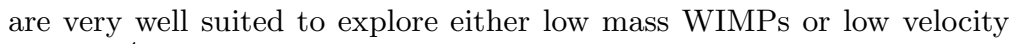
WIMPs 1 . They offer moreover the possibility of using different targets to tune up the sensitivity for different WIMP masses. Finally, they can discriminate the background (electron recoils) from nuclear recoils by collecting not only the phonon energy but also the charge (or light) produced the ionizing component of the deposited energy (hybrid detection) 10.11. The resulting background reduction permits to increase significantly the sensitivity of WIMP searches allowing to explore regions below $\sigma^{p} \sim 10^{-8}$ nbarn for medium mass $(\sim 50 \mathrm{GeV})$ WIMPs-where the neutralino configuration 12 relevant to interpret an annual modulation effect 13 is located. Although the radioactivity content of bolometers and their environment is still higher than that of HPGe, significant progresses are being accomplished.

A WIMP search with small sapphire bolometers, ROSEBUD (Rare Objects Search with Bolometers Underground), is beingerified out in the Canfranc Underground Laboratory (at 2450 m.w.e.) 14.15 , 16 with the purpose of exploring the WIMPs scattering off $\mathrm{Al}$ and $\mathrm{O}$ nuclei. It features three small sapphire bolometers (with Ge thermistors) placed in a small, mobile dilution refrigerator in the ultralow radioactivity environment of the Canfranc underground site. 


\section{Experimental set-up and performances}

The first phase of the experiment consists of two 25g (B175 and B200) and one 50g (B213) selected sapphire bolometers operating inside a small refrigerator (stick type, $6 \mathrm{~cm}$ diameter, $100 \mathrm{~cm} \mathrm{long}$ ) at $20 \mathrm{mK}$. One of the $25 \mathrm{~g}$ sapphire crystals (B200), mounted on top of the set is part of a composite bolometer ( $2 \mathrm{~g}$ of $\mathrm{LiF}$ enriched at $96 \%$ in ${ }^{6} \mathrm{Li}$ are glued to it) to monitor the thermal and fast neutron background of the laboratory. A cylinder (34.5 mm diameter and $30.8 \mathrm{~mm}$ high) in Roman lead is placed below B200 to screen the two lower sapphire absorbers (see Fig. 1) from radioactivity in the components of the dilution unit.

The shielding of the ROSEBUD experimental box has an inner part inside the dilution unit at helium bath temperature, consisting of a liner of Roman lead, $4 \mathrm{~mm}$ thick, surrounding the bolometers (to shield the intrinsic radioactivity of the $0.5 \mathrm{~mm}$ thick stainless steel walls of the inner vacuum chamber) and of a $14.4 \mathrm{~mm}$ thick high purity copper (to shield the radioactivity of the outer dewar). The ancient (2000 yr) Roman lead of the shielding has very low contamination $\left(<9 \mathrm{mBq} / \mathrm{kg}\right.$ on ${ }^{210} \mathrm{~Pb}$ and $<0.2 \mathrm{mBq} / \mathrm{kg}$ on ${ }^{238} \mathrm{U}$ and $<0.3 \mathrm{mBq} / \mathrm{kg}$ on $\left.{ }^{232} \mathrm{Th}\right)$. The external part of the shielding (at room temperature) consists of $10 \mathrm{~cm}$ of Roman lead bricks, $15 \mathrm{~cm}$ of low-activity lead (of about $100 \mathrm{yr}$ old and of 30 $\mathrm{Bq} / \mathrm{kg}$ of $\left.{ }^{210} \mathrm{~Pb}\right)$, then a $1-\mathrm{mm}$ Cadmium foil and a mu-metal screen plus a plastic box which close tightly the enceinte. A diagrammatic view of the experimental box within the ROSEBUD dilution refrigerator and the shielding set-up are depicted in Figure 1 a) and b) respectively. The experimental setup is installed within a Faraday cage in an acoustic isolation cabin, supported by an antivibration platform, as required to reach low thresholds. Slight modification of the experimental set-up have been done along the successive runs for improving the background and the energy threshold.

Power supply inside the cabin is provided by batteries and data transmission from the cabin through convenient filters is based on optical fibers. Pumps have vibration-decoupled connections.

Infrared (IR) pulses are periodically sent to the bolometers through optical fibers in order to monitor the stability of the experiment. The resulting heat pulses are useful to correct any sensitivity drift of the bolometers during the experiment. The corresponding pulses are quite similar in shape to the background or ${ }^{57}$ Co source events, but slightly faster because they are mainly produced in the thermistor, the sapphire being quite transparent to infrared photons. This is illustrated in Fig. 2 where IR pulses $(\sim 4.4 \mathrm{~V})$ are clearly distinguished from background events in the sapphire.

For absolute energy calibration of the two bolometers placed at the bottom of the cryostat during the background tests, external ${ }^{57} \mathrm{Co}$ and 
${ }^{241} \mathrm{Am}$ sources are used (See Fig. B). The three lines (59.54, 122 and $136 \mathrm{keV}$ ) allow to extrapolate (assuming linear response) the calibration to lower energies. For the upper one (B200), an internal ${ }^{241} \mathrm{Am}$ source (of $3 \mathrm{~Bq}$ of activity) has been used. The alpha emission (5.5 MeV) has been degraded to $3 \mathrm{MeV}$ by mylar foils in order to leave clean the region where the neutron signal (above the $\mathrm{Q}$-value of the ${ }^{6} \mathrm{Li}(\mathrm{n}, \alpha)$ reaction, $4.78 \mathrm{MeV}$ ) should be found.

The bolometers are suspended inside an OFHC copper frame (coupled to the mixing chamber of the dilution refrigerator) by Kevlar wires tensioned to drive the mechanical resonant frequency to the highest possible value (above $400 \mathrm{~Hz}$ ). The refrigerator operating point was just below $20 \mathrm{mK}$ (mixing chamber temperature) and the bolometers were working at $28 \mathrm{mK}$ with a bias of $100-150 \mathrm{mV}$ on $40 \mathrm{M} \Omega$ load resistors. The first tests in Canfranc have shown that microphonic and electronic noise level is quite good, about $2 \mathrm{nV} / \mathrm{Hz}^{1 / 2}$ at low frequency $(30 \mathrm{~Hz})$ on bolometer B175.

The bolometers-yere tested previously in Paris (IAS) showing very good performances 14 . (FWHM energy resolution of $\Gamma(1.5 \mathrm{keV})=120 \mathrm{eV}$ and energy threshold around $300 \mathrm{eV}$ ). Typical sensitivities obtained (in Canfranc) are in the range of $0.3-1 \mu \mathrm{V} / \mathrm{keV}$. Overall resolutions of 3.2 and $6.5 \mathrm{keV}$ FWHM were typically obtained with B213 and B175, respectively, at $122 \mathrm{keV}$. With B175, $2.8 \mathrm{keV}$ FWHM was obtained at 59.5 $\mathrm{keV}$. Typical pulses have decay and rise times between 2-4 ms and 600$1300 \mu$ s, respectively. A low energy background pulse in bolometer B213 can be seen in Fig. A. It corresponds to an energy of about $2 \mathrm{keV}$.

Radiopurity measurements of some of the ROSEBUD internal components (NTD sensors, sapphire crystals, copper frames...) were carried out at the underground laboratories of Modane (LSM) and Canfranc using ultrałpw background germanium detectors and the results reported in Ref. 15. The substitution of the set-up components with radioactive impurities by other cleaner components would likely make them decrease down to levels acceptable for the first phase of the experiment, as it has actually happened in the runnings reported in the next paragraph. The upper limiton the total background produded by those impurities was estimated 15 to be about 5 counts. $\mathrm{keV}^{-1} \cdot \mathrm{kg}^{-1}$. day ${ }^{-1}$ at $10 \mathrm{keV}$. This rate is still one to two ordeffof magnitude worse than that obtained in good germanium detectors 10.

\section{Results and prospects}

ROSEBUD has performed a series of short runnings in different conditions (of a duration of a few days each) to optimize the background, minimize microphonics and improve the performances of the bolometers, before going to larger running time. 
In a first testing measurement in the shielding configuration described above a background as large as 120 counts $/ \mathrm{keV} / \mathrm{kg} /$ day around $100 \mathrm{keV}$ was obtained (Run 1). To understand and reduce this background, several components of the cryostat were measured in the ultralow background HPGe testing bench at Canfranc. After subsequent modifications in the cryostat, like the replacement of the old multilayer glass fiber insulation and the reduction of the charcoal mass, a background level of about 30 counts $/ \mathrm{keV} / \mathrm{kg} /$ day around $100 \mathrm{keV}$ was obtained in bolometers B175 and B213 (Run 2). Further reduction of the internal background was achieved by replacing some other internal pieces of the cryostat and removing completely the coconut charcoal, which was replaced by artificial carbon tissue (Actitex), and adding a further in lead shielding ( $15 \mathrm{~cm}$ high, $15 \mathrm{~cm}$ external diameter and $5 \mathrm{~mm}$ thick) 16 . The resulting background was 15 counts/(keV kg day) around $100 \mathrm{keV}$ (Run 3). The next efforts were focused in eliminating possible radon contaminations inside the nitrogen reservoir of the cryostat as well as in changes in the inner configuration, in particular, removing the bottom bolometer B175 and putting instead an additional archaeological lead cylinder $4 \mathrm{~cm}$ thick. A short running to test the progress of this reduction shows a background of $\sim 18$ counts/( $\mathrm{keV} \mathrm{kg}$ day) in the region from 25 to $50 \mathrm{keV}$, of $\sim 11$ counts/(keV kg day) between 50 and 100 $\mathrm{keV}$ and $\sim 7$ counts/(keV kg day) between 100 and $150 \mathrm{keV}$ (Run 4). The four successive background spectra obtained in the corresponding set-up configurations enumerated above are shown in Fig. 5. A rise time cut has been applied to eliminate background interactions in the NTD thermistor (see Fig. 2p. By taking the spectrum of Run 4 (see Fig. 6) - corresponding to an exposure of 2.1 days with bolometer B213 of 50 $\mathrm{g}$ of $\mathrm{Al}_{2} \mathrm{O}_{3}$ - obtained with a conservative rise time cut - applied only down to $25 \mathrm{keV}$ - a $90 \%$ C.L. exclusion plot $\sigma(\mathrm{m})$ has been derived for spin-independent interaction with the conservative assumption of a 2$\mathrm{keV}$ low energy resolution and a $2-\mathrm{keV}$ threshold. The exclusion plots have been derived by requiring the predicted WIMP signal in the $2-\mathrm{keV}$ energy bin from 2 to $100 \mathrm{keV}$ to be less than or equal to the $90 \%$ C.L. upper limit of the (Poisson) background counts recorded there. The derivation of the interaction rate signal supposes that the WIMPs form an isotropic, isothermal, non-rotating halo of density $\rho=0.3 \mathrm{GeV} / \mathrm{cm}^{3}$, have a Maxwellian velocity distribution with $\mathrm{v}_{\mathrm{rms}}=270 \mathrm{~km} / \mathrm{s}$ (with an upper cut corresponding to an escape velocity of $650 \mathrm{~km} / \mathrm{s}$ ), and have a relative Earth-halo velocity of $\mathrm{v}_{\mathrm{r}}=230 \mathrm{~km} / \mathrm{s}$. The quenching factor (nuclear versus electron recoil efficiency) has been taken unity $-\rho .98 \pm 0.05$ for sapphire bolometers measured at $100 \mathrm{keV}$ recoil energy 21). A Fermi nuclear form factor has been used. Fig. 0 shows the contour line of this exclusion cerppored with that obtained with Ge (Gefpmbined from published data $4,1,19$ and the more recent COSME-2 318 and IGEX 
3.18) and NaI (DAMA-0) detectors 20 The region singled out by the DAMA modulation effect is also shown 13 .

Fully understanding and significantly improving the background is the current objective of ROSEBUD. With such purpose, measurements of the radiopurity of individual components proceed and their removal done when needed. Assuming a flat background of 1 count $\mathrm{keV}^{-1} \mathrm{~kg}^{-1}$ day $^{-1}$, a threshold energy of $300 \mathrm{eV}$ and a FWHM energy resolution of $100 \mathrm{eV}$ in the low energy repion (nominal values obtained for the bolometer B213 in the Paris tests 3 ), a projection of the ROSEBUD sensitivity is shown in Fig. 8 in terms of the WIMP-nucleon spin-independent interaction exclusion plot. To derive such plot, the chosen energy bin has been from 300 to $800 \mathrm{eV}$. Projected exclusion contours $\sigma(\mathrm{m})$ are shown for two typical cases: $50 \mathrm{~g}$ of $\mathrm{Al}_{2} \mathrm{O}_{3}$ operating 30 days and operating along one year. The area enclosed by the dotted line represents the region for the neutralino-nucleon cross section versus mass allowed by an unconstrained Minimal Supersymmetric Standard Model(MSSM) where usual GUT relations among gaugino masses are relaxed2. The exclusion plots have been renormalized to the neutralino-nucleon cross section and, for comparison, the most recent 9 Ge combined bound is also shown. Assuming the mentioned projected performances, ROSEBUD will allow to explore neutralino configurations corresponding to masses well below 10 $\mathrm{GeV}$.

The second phase of the ROSEBUD program will deal with three different bolometers: the current B213 sapphire bolometer of $50 \mathrm{~g}$, a new 50 g sapphire with cleaner components, and a germanium bolometer of 67 $\mathrm{g}$, operating together to investigate the target dependence of the WIMP rate, provided that a reliable understanding of their background peculiarities be achieved. As a further step in this line of research, the use of two medium-size bolometers, one of $200 \mathrm{~g}$ of $\mathrm{Al}_{2} \mathrm{O}_{3}$ and the other a double luminiscent bolometer ( $\mathrm{BGO}$ or $\mathrm{CaWO}_{4}$ ) is envisaged. Comparison of the spectra of these different mass number bolometers will help to understand the background and hopefully explore (within the same provisions) the mass number dependence of the signal rate as a genuine characteristic of WIMPs.

\section{Acknowledgements}

This work has been supported by the Spanish CICYT (contract AEN991033), the French CNRS/INSU (MANOLIA program) and by the EU Network Contract ERB-FMRX-CT-98-0167. We greatly acknowledge the help of J.P. Moalic, P. Pari and O. Testard for the cryogenics design. We are also endebted to C.Goldbach and G. Nollez for their contribution to the radioassays of various materials in the Modane Underground Laboratory (Frejus), as well as for fruitful discussions. We also thank J.L. 
Picolo, P. Cassette (LNHB) and P. Bouisset (LMRE) for their radioactive measurements of some inner components. N.Coron, G.Dambier and P. de Marcillac acknowledge financial support of the DGA-CAI Europa Program.

\section{References}

1. G. Jungman, M. Kamionkowski and K. Griest, Phys. Rep. 267 (1996) 195.

2. A. Gabutti et al., Astrop. Phys. 6 (1996) 1.

3. A. Morales, "Direct Detection of WIMP Dark Matter" (astro-ph/9912554). Review Talk at the TAUP 99 Workshop, College de France, Paris. To be published in Nucl. Phys. B (Proc. Suppl.) 2000.

4. T. Damour and L.M. Krauss, Phys. Rev. Lett. 81 (1998) 5726.

5. A. Bottino, F. Donato, N. Fornengo and S. Scopel, preprint DFTT1/2000, hep-ph/0001309.

6. N. Booth, B. Cabrera and E. Fiorini, Ann. Rev. of Nucl. Sci. 46 (1996) 471.

7. J. Morales et al., Nucl. Instrum. \& Meth. A321 (1992) 410 and E. García et al., Phys. Rev. D51 (1995) 1458.

8. D. Reusser et al., Phys. Lett. B255 (1991) 143.

9. A. Morales et al., "New Constraints on WIMPs from the Canfranc IGEX Dark Matter Search", hep-ex/0002053 (21 February 2000).

10. G. Chardin et al. "Status of the EDELWEISS experiment", prestented at TAUP 99, Collège de France, Paris, September 1999. To appear in Nucl. Phys. B (Proc. Suppl.)

11. R. Abusaidi et al, "Exclusion limits on the WIMP-nucleon crosssection from the CDMS", astro-ph/0002471 (25 February 2000) and references therein.

12. A. Bottino et al., Phys. Lett. B243 (1998) 109.

13. R. Bernabei et al., Phys. Lett. B450 (1999) 448, ROM2F/2000/01, January 2000.

14. C. Bobin et al., Nucl.Phys. B (Proc. Suppl.) 70 (1999) 90.

15. S. Cebrián et al., Astrop. Phys. 10 (1999) 361.

16. S. Cebrián et al., to appear in Nucl. Instr. \& Meth.A (2000) (LTD8 Conference), and in Nucl. Phys. B (Proc. Suppl.) (2000) (TAUP 99 Workshop).

17. L. Baudis et al., Phys. Rev. D59 (1999) 022001.

18. S. Cebrián et al., "Dark Matter Searches at Canfranc", to appear in the New Journal of Physics (2000).

19. A.K. Drukier et al., Nucl. Phys. B. (Proc. Suppl) 28A (1992) 293.

20. R. Bernabei et al., Phys. Lett. B379 (1996) 299.

21. J.W. Zhou et al, Nucl. Instr. \& Methods A349 (1994) 225. 


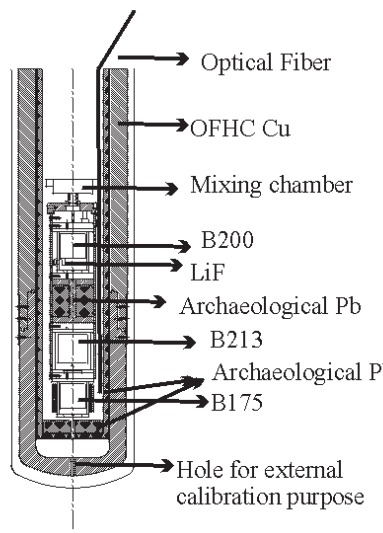

(a)

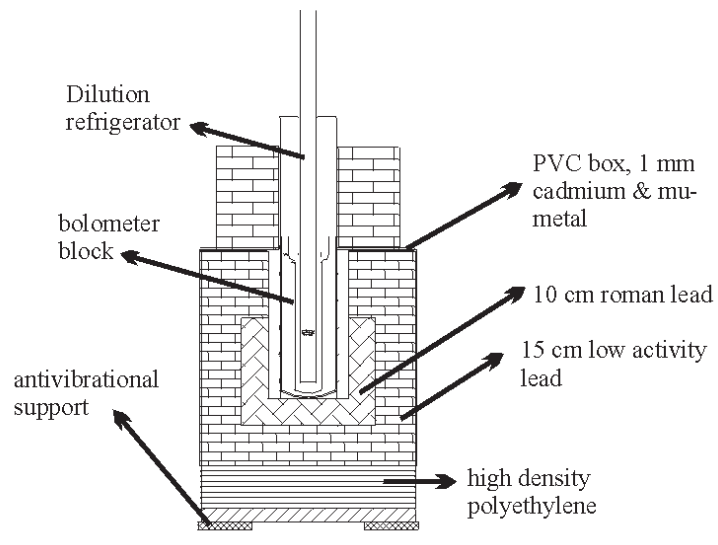

(b)

Figure 1: (a) Schematic view of the bolometer block in the first ROSEBUD run at Canfranc. See text for explanation. (b) View of ROSEBUD set-up. 

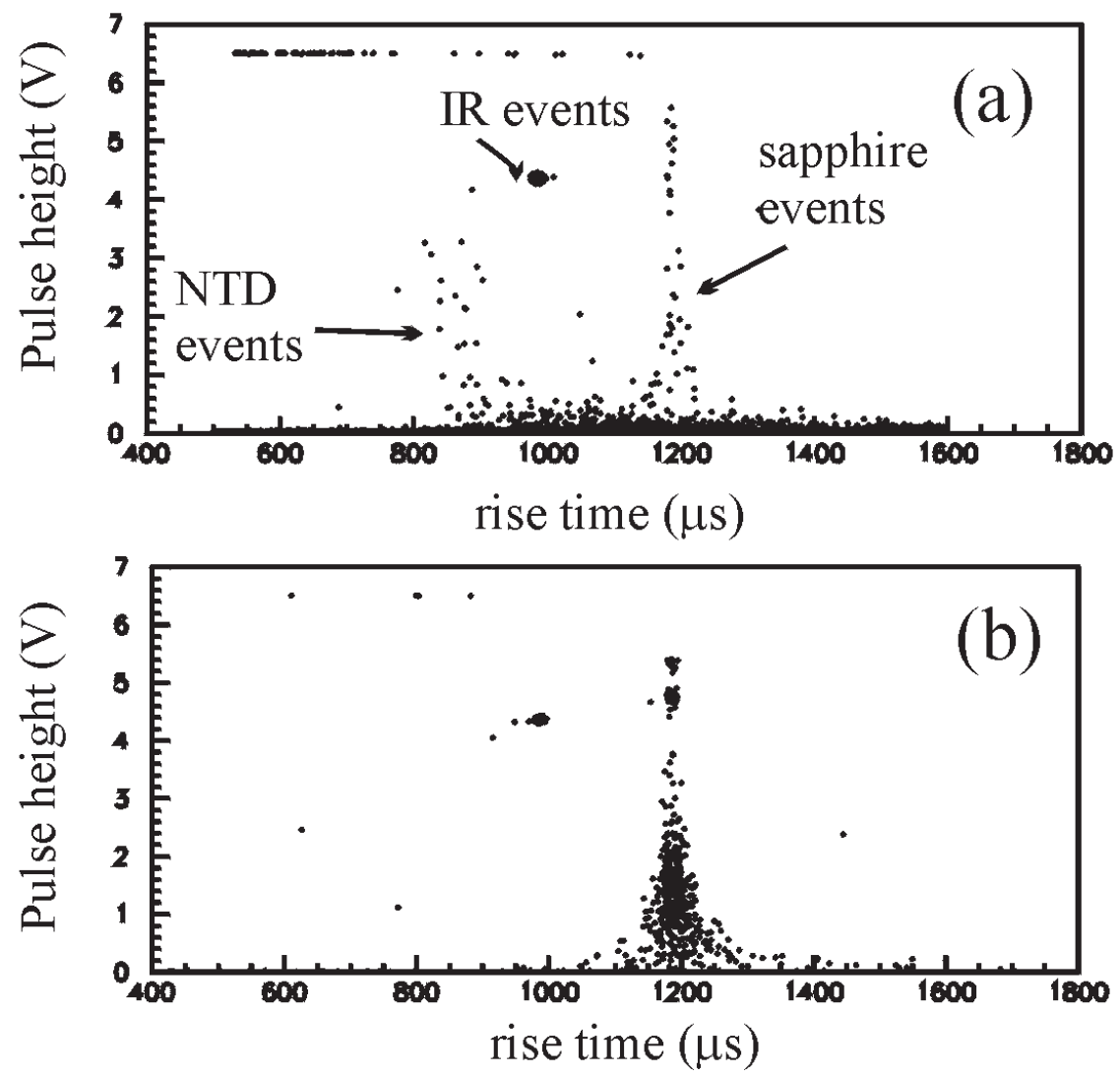

Figure 2: Rise time discrimination between IR pulses $(\sim 4 \mathrm{~V}$ and $\sim 1000 \mu \mathrm{s})$ and background events in the sapphire $(\sim 1200 \mu \mathrm{s})$ recorded on bolometer B213 along a backgound measurement (a) and a ${ }^{57}$ Co calibration (b). Events having low risetime in Fig. a can be attributed to background interactions in the NTD thermistor. 

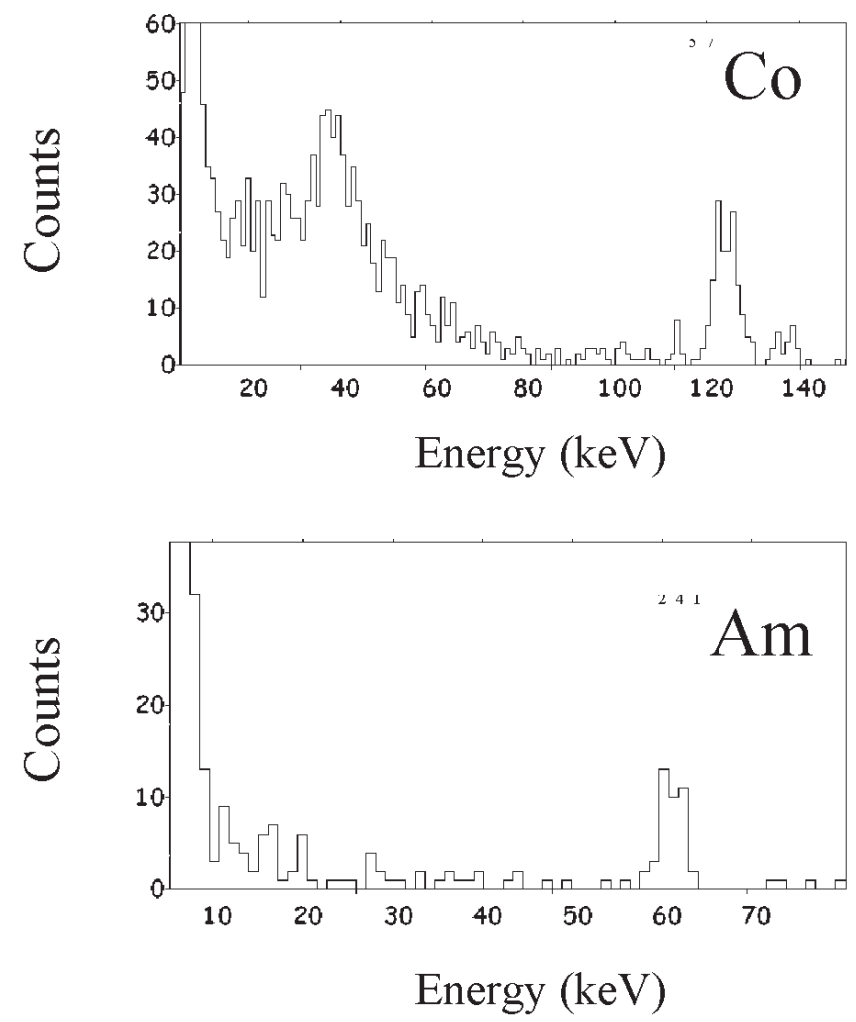

Figure 3: Typical calibration spectra from ${ }^{57} \mathrm{Co}$ and ${ }^{241} \mathrm{Am}$ sources in bolometer B175. 


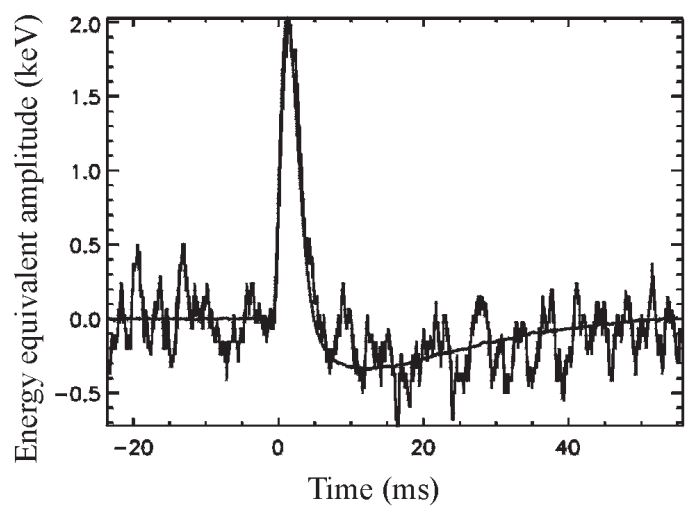

Figure 4: A pulse of about $2 \mathrm{keV}$ obtained at Canfranc with bolometer B213. A normalized $100 \mathrm{keV}$ event is overplotted.

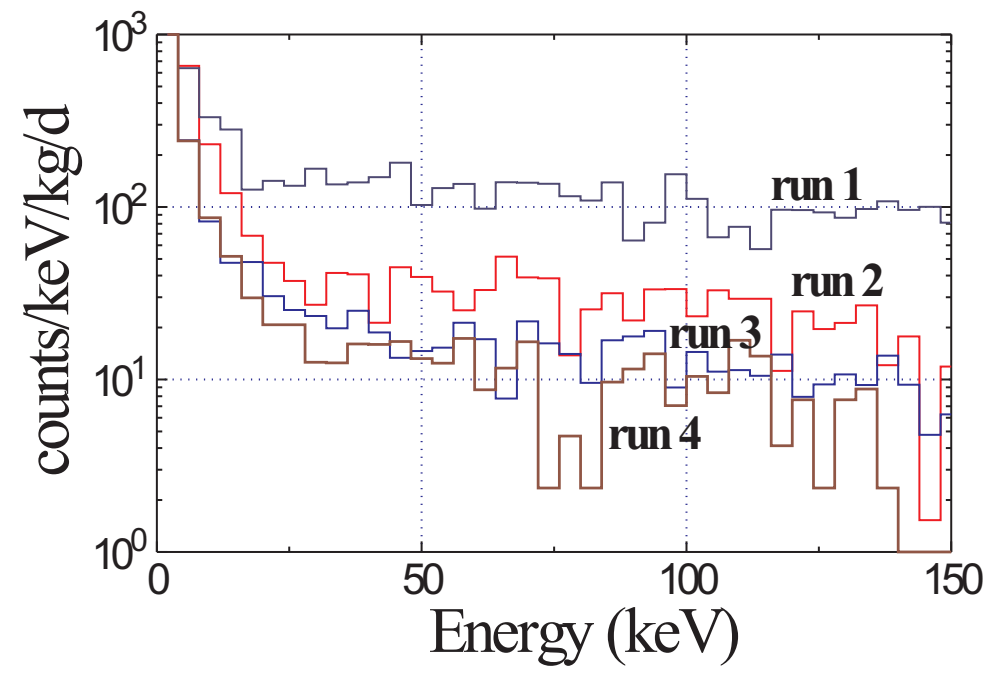

Figure 5: The four successive background spectra of ROSEBUD obtained after replacement of several components. 


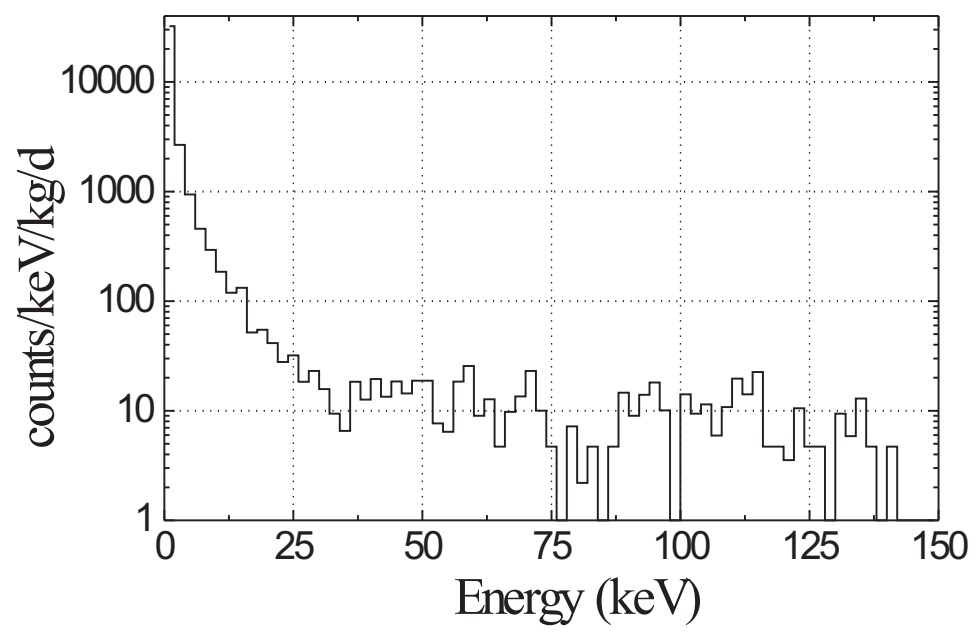

Figure 6: Spectrum of the last running (Run 4) corresponding to 2.1 days of exposure of bolometer B213 of $50 \mathrm{~g}$. Conservatively, the cut in rise time has been applied only down to $25 \mathrm{keV}$. 


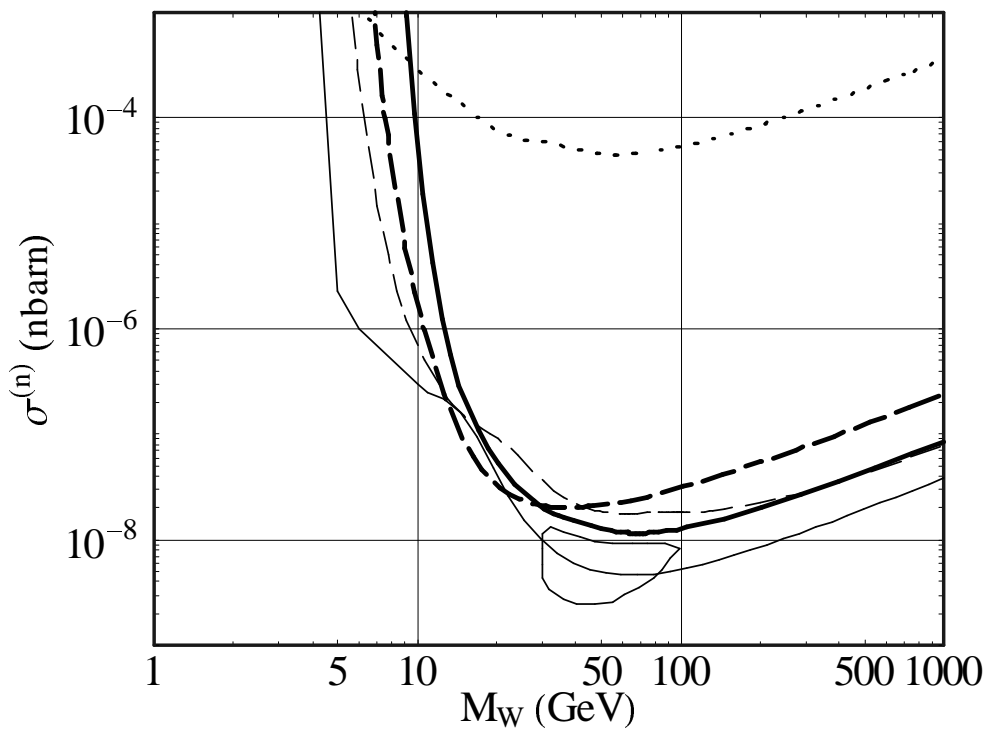

Figure 7: Exclusion plot (dotted line) cross-section versus WIMP mass for spin-independent WIMP-nucleon interaction at the $90 \%$ C.L. derived from the spectrum of Figure 6 . Other exclusion limits obtained with Ge and NaI detectors are also shown: The Ge combined bound from published data (thin dashed line) and the more recent COSME-2 (thick dashed line) and IGEX (thick solid line) results are shown together with the DAMA (NaI-0) exclusion contour (thin solid line) and the DAMA (NaI-1,-2,-3,-4) annual modulation region (closed contour). 


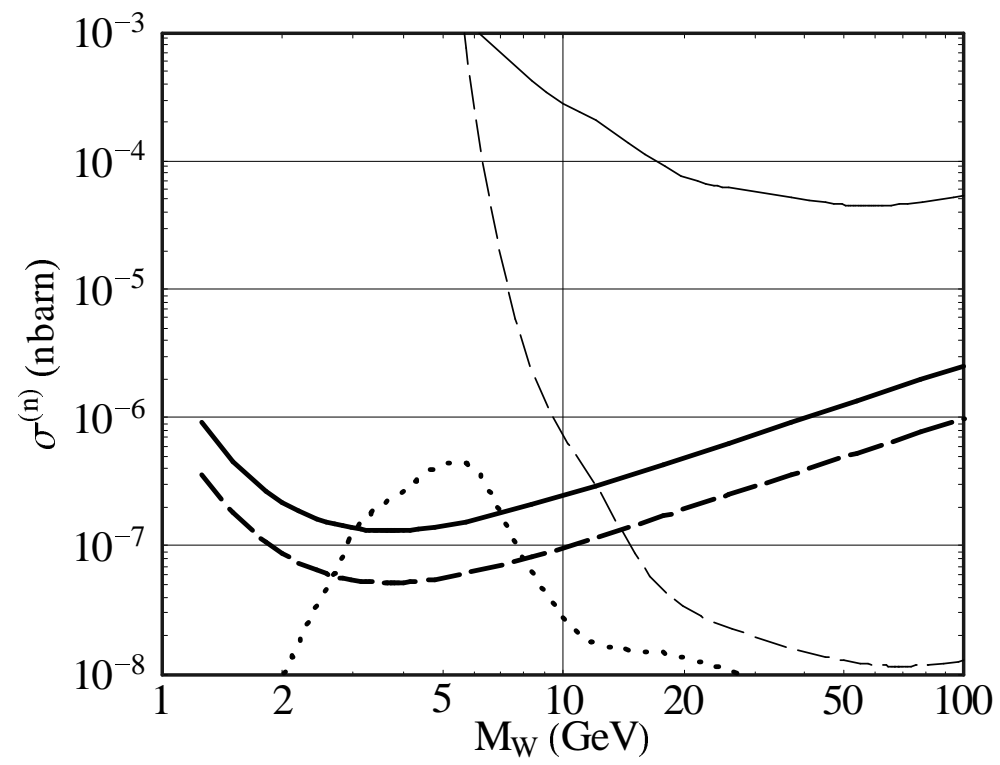

Figure 8: Projected $\sigma(\mathrm{m})$ exlcusion plots of the ROSEBUD experiment for spin-independent coupling (assuming a flat background of $1 \mathrm{c} / \mathrm{keV} \mathrm{kg} \mathrm{day,} \mathrm{a} \mathrm{threshold} \mathrm{of} 300 \mathrm{eV}$ and an energy resolution of $100 \mathrm{eV}$ ), for the cases: $50 \mathrm{~g}$ of sapphire operating 30 days (thick solid line) and along one year (thick dashed line) compared with the unconstrained MSSM allowed region (dotted line). The current ROSEBUD result (thin solid line) and the total Ge-combined bound (thin dashed line) are also shown. 